\begin{tabular}{|l|l|}
\hline BJPsych & BJPsych Open (2021) \\
\hline 0 (0) & 7(S1), S1-S356 \\
\hline
\end{tabular}

\section{BJPsych Open}

cambridge.org/bjo

\section{Abstracts}

Cite this article: (2021). Abstracts from the RCPsych Virtual International Congress 2021, 21-24 June. BJPsych Open 7(S1), S1-S356

\section{Abstracts from the RCPsych Virtual International Congress 2021, 21-24 June}

The submitted Abstracts from Posters presented at the RCPsych International Congress 2021, 21-24 June are published as a special supplement to BJPsych Open.

\section{Peer review process}

Abstracts were reviewed by the RCPsych Academic Faculty rather than by the standard BJPsych Open peer review process and should not be quoted as peer-reviewed by BJPsych Open in any subsequent publication.

\section{Disclaimer}

The abstracts have been published using author-supplied copy, with only minor editing made to correct spelling, style and format where appropriate. No responsibility is assumed for any claims, instruction or methods contained in the abstracts and it is recommended that these be verified independently.

\section{Rapid-Fire Poster Presentations}

\section{Selected from the top scoring abstracts from all categories. Arranged by presenting author surname.}

\section{Evaluation of a mental health first aid workshop for healthcare professionals \\ Sara Abou Sherif ${ }^{\star}$ and Sachin Patel}

Hammersmith \& Fulham Liaison Psychiatry, Charing Cross Hospital, Imperial College NHS Healthcare Trust, London UK

${ }^{\star}$ Corresponding author.

doi: 10.1192/bjo.2021.67

Aims. Annually, 1 in 4 people in the UK will experience a mental health problem. Alongside the approach of increasing awareness of the issue amongst the general population, there is a drive to deliver training and education on the recognition and management of mental health crises. Limited resources exist to aid healthcare professionals in delivering mental health first aid (MHFA), with the vast majority focussing on lengthy training courses. Small group problem-based learning (PBL) is utilised widely in medical education and this modality offers advantages in deliverability, audience participation and experiential learning. Our aim was to deliver and explore the effectiveness of a PBL MHFA workshop to various healthcare professionals.

Method. As part of an Emergency Medicine Mental Health Education day, we delivered four 30-minute PBL MHFA workshops. These involved an introduction to MHFA, followed by an interactive discussion of 4 mental health simulated cases, whereby participants anonymously answered a range of questions using the web-based platform Mentimeter. We devised a simple MHFA A,B,C,D,E acronym to bring structure to problem solving. Pre- and post-workshop questionnaires were used to assess outcomes using Likert scales to measure various aspects of MHFA ( $1=$ strongly disagree and $5=$ strongly agree). Statistical significance was calculated using T-Test with $\mathrm{P}<0.05$ defining statistical significance.

Result. A total of 28 professionals attended the workshops, 20 (72\%) completed both the pre and post workshop questionnaire. $19(76 \%)$ were nurses (5 Registered Mental Health Nurses and 14 Registered General Nurses), 3 (12\%) were doctors, 2 (8\%) were HCA's and 1 was a policeman. 15 (75\%) of the participants reported historically having had the need to deliver MHFA but only 3 (15\%) had previously received training. After the workshop, participants reported significantly increased understanding [3.0 to $4.3(\mathrm{p}<0.05)$ ] and confidence in delivering MHFA [3.05 to $4.30(\mathrm{p}<0.05)$ ]. There was significantly improved confidence in assessing risk [3.03 to 4.05], calling for appropriate help [3.45 to 4.35] and de-escalation techniques [3.05 to 4.15].

Conclusion. To our knowledge this is the first mini PBL-based MHFA workshop. We have demonstrated that the PBL workshop setup is an effective means to deliver training on MHFA. We recognise the importance of MHFA training reaching a larger audience and its potential value if incorporated into national healthcare training programmes and made available to the general public. 
Prevalence of depression, anxiety and stress disorders among medical students in Alexandria Faculty of Medicine during COVID-19 pandemic

Hanan M. Hemead ${ }^{1}$, Sarah Hemead ${ }^{1}$, Ahmed Shaheen ${ }^{1}$, Nour Shaheen ${ }^{1}$, Ehab Elrewany ${ }^{2}$ and Hesham Adel Sheshtawy ${ }^{1, *}$

${ }^{1}$ Alexandria Faculty of Medicine and ${ }^{2}$ High Institute of Public Health, Alexandria University

${ }^{*}$ Corresponding author.

doi: 10.1192/bjo.2021.68

Aims. We aimed to assess the impact of the current pandemic on the mental well-being of undergraduate medical students of Alexandria Faculty of Medicine, Egypt.

Method. We designed a structured anonymous online questionnaire and encourage students to fill it in on social platforms. The questionnaire is composed of seven parts, each one includes multiple choice questions aimed to measure the impact of the pandemic on different aspect of daily activities namely: academic performance, social and family relationship, eating and smoking habits, sleep pattern, physical activity and the Depression, anxiety and stress scale (DASS-21). The last part was an open question where participants can state their comments about the experiences during the quarantine and how they affected their mental health. Result. A total of 1181 students from the six academic grades responded. Females and students in the third academic year showed the highest prevalence of depression, anxiety and stress. Overall, most respondents reported that the current pandemic had negative impacts on their academic performance $(71 \%)$ and social relationship $(67.5 \%)$. The majority of the students stated that they became less physically active $(74.6 \%)$ and $52.2 \%$ experienced a weight gain. Despite that $60 \%$ of the studied population rated their sleeping quality as "very good" and "fairly good", $45.3 \%$ and $39.6 \%$ suffered from increased sleeping hours and disturbed sleep respectively. Based on students' responses of the DASS-21, over half of the participants (62.2\%) were experiencing moderate to extremely severe stress and over 33\% were consistent with symptoms of extremely severe anxiety. In respect of depression, nearly half of the sample (46.4\%) can be described as having extremely severe depression according to the cut-off points of the DASS-21.

Conclusion. The current pandemic has increased the challenges and burdens on undergraduate medical students. These impacts can be more profound in developing countries such as Egypt. The levels of psychiatric symptoms are alarming compared to previous local and international studies. These unprecedented consequences should be addressed promptly through students' counselling and psychiatric assistance. To date, this is the largest psychiatric and survey-based study conducted on Alexandria Faculty of Medicine.

Financial disclosure: The study was not funded by any organization, the authors did not receive any financial aids.

\section{Post traumatic growth during COVID-19: unity in diversity}

Meena Afzal Lakha ${ }^{1, *}$, Anindya Bhowmik², Sneha Bisht ${ }^{3}$, Suzani Shrestha ${ }^{4}$, Kantappa Gajanan ${ }^{5}$ and Samir Shah ${ }^{6}$

${ }^{1}$ Wrightington Wigan \& Leigh NHS Foundation Trust; ${ }^{2}$ London Northwest University Healthcare Trust; ${ }^{3}$ Cambridge University Hospital NHS Foundation Trust; ${ }^{4}$ Oxford University Hospital NHS Foundation Trust; ${ }^{5}$ The Christie NHS Foundation Trust and ${ }^{6}$ Priory Hospital Altrincham

${ }^{*}$ Corresponding author.

doi: 10.1192/bjo.2021.69
Aims. This poster reflects how the experience of staying with people of diverse nations and cultural background helped the stranded IMGs cope with this agony in a foreign land during an unprecedented tumultuous situation. The aim is to show that despite diversity among people, the hard times made them unite and overcome countless difficulties.

Background. The COVID 19 pandemic has been a period of global health crisis and has exponentially affected mental health issues in the world population. In these difficult times, several International Medical Graduates (IMGs), who had come to the UK to attend their PLAB exams, were left stranded as the exams were postponed, flights cancelled and borders sealed. Faced with huge uncertainty their mental health was of great concern.

At this time the British Association of Physicians of Indian Origin (BAPIO) came forward to help this cohort of stranded doctors in terms of accommodation, finances, mental health support, preparation for exams to the extent of liaising with General Medical Council (GMC) and Home Office. The virtual support group provided a platform for IMGs from different nations and cultures to get in touch with each other helping overcome mental burden and stress.

The stories presented in the poster show how unity in diversity helped these young doctors deal with mental trauma amidst the Pandemic.

Method. 276 doctors from 27 countries were looked after by BAPIO. From those excerpts taken from 26 IMGs, personal narratives was used as a method for qualitative assessment.

The percentage of IMGs clearing their exams and getting jobs in the NHS has been used for quantitative assessment.

Result. Qualitative: The personal narratives of the IMGs show how they were positively impacted by staying together albeit different nationalities and cultural background.

Quantitative: A total of 21 IMGs out of the 26 cleared their PLAB 2 exams and got registration under General Medical Council giving a percentage of $81.7 \%$. 20 IMGs have successfully joined the NHS in various posts giving a job success rate of $95.2 \%$. Conclusion. The experience of living and sharing housings with people from different nationalities, has increased appreciation and also prepared them to work in the NHS which has a diverse work force. This learning experience has been integral for all of us in shaping our life in the UK making everyone more compassionate.

\section{Attitudes of medical students towards choosing psychiatry as a career}

Mohammad Ahmad ${ }^{1, *}$, Marwan Dabbagh ${ }^{2}$, Alawwab Dabaliz ${ }^{3}$ and Akef Obeidat ${ }^{2}$

${ }^{1}$ Barnet Enfield and Haringey MH NHS Trust, Lancashire Care NHS Foundation Trust; ${ }^{2}$ Alfaisal University and ${ }^{3}$ University Hospitals, Cleveland Medical Center ${ }^{*}$ Corresponding author.

doi: 10.1192/bjo.2021.70

Aims. Our aim is to study factors influencing attitudes of medical students towards pursuing Psychiatry as a career. We hypothesise that the minimal exposure and/or importance given to Psychiatry during medical school is insufficient to let a student truly experience and appreciate the specialty.

Background. Studies report an annual decline in Psychiatry Trainees in many parts of the world. This deficiency is projected to create gaps between mental health service needs and providers. Studies have also explored the crisis in recruitment and the 